\title{
YIPF5 mutations cause neonatal diabetes and microcephaly: progress for precision medicine and mechanistic understanding
}

\author{
Toni I. Pollin and Simeon I. Taylor \\ Division of Endocrinology, Diabetes, and Nutrition, Department of Medicine, University of Maryland School of Medicine, Baltimore, Maryland, USA
}

\begin{abstract}
Identifying genes that result in monogenic diabetes can provide insights that can build a scientific foundation for precision medicine. At present, nearly $20 \%$ of neonatal diabetes cases have unknown causes. In this issue of the $J C I$, De Franco and Lytrivi et al. sequenced the genome of two probands with a rare neonatal diabetes subtype that also associated with microcephaly and epilepsy. The authors revealed mutations in the YIPF5 gene. YIPF5 resides in the Golgi apparatus and is thought to play a critical role in vesicular trafficking. Notably, disrupting YIPF5 in $\beta$ cell-based models induced ER stress signaling and resulted in the accumulation of intracellular proinsulin. We believe that utilizing registries and biobanks to reveal other monogenic atypical forms of diabetes is an important approach to gaining insight and suggest that an insulin sensitizer may alleviate ER stress associated with YIPF5 disruption by decreasing the demand for insulin secretion.
\end{abstract}

\section{Monogenic diabetes genes}

Precision medicine for diabetes is often said to be on the horizon. However, as noted in the recent joint American Diabetes Association (ADA)/European Association for the Study of Diabetes (EASD) Precision Medicine in Diabetes Initiative (PMDI) consensus report (1), in some instances the horizon stretches back several decades. The existence of monogenic forms of diabetes has long been known. In 1974, Tattersall noted "mild familial diabetes with dominant inheritance" (2) in some families, and shortly thereafter Tattersall together with Fajans coined the term "maturity onset diabetes of the young (MODY)." MODY is distinguished clinically from classical juvenile-onset diabetes (now known as type 1 diabetes) by a lower degree of severity and the lack of a requirement for insulin treatment. The age of onset sets MODY apart from maturity-onset diabetes (now known as type 2 diabetes). MODY's distinct Mendelian inheritance pattern sets it apart from both types (3).

The first three MODY genes were identified as GCK (MODY2) in 1992 (4), HNF4A (MODY1) in 1996 (5), and HNF1A (MODY3) in 1996 (6). A separate stream of research identified several monogenic diabetes syndromes characterized by severe insulin resistance. Although generalized lipodystrophy was described in the 1950s (7, $8)$, it would take another forty-plus years to identity two disease genes: AGPAT2 (9) and BSCL2 (10). In addition, several syndromes of insulin-resistant diabetes syndromes

\section{Related Article: p. 6338}

Conflict of interest: TIP receives partial research funding from a grant to the University of Maryland, Baltimore, by the Regeneron Genetics Center. SIT serves as a paid consultant to lonis Pharmaceuticals and is listed as an inventor on a methods of use patent, titled "Use of leptin for treating human lipoatrophy and method of determining predisposition to said treatment" (US 7,183,254 B2)

Copyright: ( 2020, American Society for Clinical Investigation.

Reference information: / Clin Invest. 2020;130(12):6228-6231. https://doi.org/10.1172/JCl142364

were described in the 1970s $(11,12)$, and shown in the 1980s to be caused by mutations in the insulin receptor gene (13-15).

\section{Precision medicine opportunities}

As emphasized by Hattersley and Patel (16), precision medicine has at least three important benefits: (a) optimizing choice of medications, (b) enhanced ability to predict patients' clinical courses, and (c) enabling early diagnosis through genetic technology. For example, nine years after the identification of HNF1A as the MODY3 gene, Hattersley and colleagues reported that individuals harboring $H N F 1 A$ mutations and previously diagnosed with type 1 diabetes could switch from insulin injections to low-dose oral sulfonylureas without loss of glycemic control (17). As this group at the University of Exeter led the way in implementation, they and others continued to discover monogenic diabetes genes. They made another breakthrough discovery in 2004 by finding that 10 of 29 patients with the rare condition of neonatal diabetes had activating mutations in the KCNJ11 gene encoding the Kir6.2 subunit of the ATP-sensitive potassium channel (18). Just two years later they established that high-dose oral sulfonylureas, instead of insulin injections, could successfully treat most of the patients with KCNJ11 mutations (19).

Fast-forward to today. De Franco and Lytrivi et al. (20) note in this issue of the JCI that, prior to the current work, 30 neonatal diabetes genes had been identified - accounting for $82 \%$ of cases. Variants in some of these genes cause extrapancreatic syndromic features, including neurological features with 11 of the genes. Identification of these rarer neonatal subtypes yields precision medicine opportunities as well; for example, FOXP3 mutations in neonatal diabetes reveal an underlying polyendocrinopathy in which early treat- 
ment with a bone marrow transplant can prevent otherwise often fatal gastrointestinal enteropathy (21). Precision medicine has also led to an innovative treatment for generalized lipodystrophy. Metreleptin provides life-changing efficacy to patients with generalized lipodystrophy (22) and has received regulatory approval as a new drug specifically indicated to treat that orphan disease.

The Exeter group has a strong diagnostic program for monogenic diabetes. Patients evaluated throughout the United Kingdom and worldwide undergo next-generation sequencing on a growing panel currently containing 71 known or putative monogenic diabetes genes, where a subset is analyzed guided by clinical information. A well-curated list of these genes is provided on the DiabetesGenes website (https://www.diabetesgenes.org/). This diagnostic program is currently the most cost-effective method for achieving a clinical genetic diagnosis and optimally captures the specific genes involved. However, this routine approach to clinical diagnosis does not allow for the discovery of variants in novel genes. Nearly all cases of neonatal diabetes are monogenic rather than type 1 , but the cause remains unknown in approximately $18 \%$ of cases. This remaining $18 \%$ provides a strong rationale to conduct exome sequencing in panel-negative cases. When studying known genes, it is already challenging to distinguish disease-causing from benign variation. Because of differences in how genetic variants had been classified, even for well-established causal genes, standards and guidelines for interpretation of sequence variants were developed and published by the American College of Medical Genetics (ACMG)/ Association of Molecular Pathologists (AMP) in the United States (23) and the Association for Clinical Genomic Science Best Practice Guidelines in the United Kingdom (https://www.acgs.uk. com/quality/best-practice-guidelines/). To further improve the accuracy and consistency of monogenic diabetes variant classification in particular, and to assure centralized availability of the knowledge, the international ClinGen (Clinical Genome Resource, https://clinicalgenome.org/) Monogenic Diabetes Expert Panel (MDEP) was convened in 2017. This group, based at the University of Maryland School of Medicine, brings experts together to pool clinical data and to develop and implement disease- as well as gene-specific variant classification rules (24).

Exome sequencing is challenging because of the volume of data generated and consequent magnified difficulty of distinguishing benign from causal variation. The risk of false positives is high. One must go about it in a both systematic and open-minded, exploratory manner. It is helpful to define a specific phenotype, study multiple affected individuals, and suspect a specific inheritance pattern. In De Franco and Lytrivi et al. (20), the authors met these requirements by sequencing two unrelated individuals with the specific constellation of neonatal diabetes, seizures, and severe microcephaly. Both were born to first-cousin pairs, increasing the likelihood of autosomal recessive inheritance due to homozygous inheritance in each case of a variant shared by the related parents. This approach allowed the authors to elegantly narrow down the number of genes of interest to exactly one, YIPF5, in which both patients were homozygous for rare (not found in GnomAD, the largest public genomic variant database) coding variants for which their parents were heterozygous (20). This finding was promising but not sufficient evidence since the overlap could have occurred by chance. However, sequencing the gene in 187 additional individuals yielded three more probands and one affected sibling with the appropriate genotype, along with a similar specific syndromic phenotype (epilepsy and microcephaly in addition to diabetes) to the original two probands. The researchers identified homozygosity for five distinct rare coding variants, providing strong genetic evidence that YIPF5 was indeed the disease gene in these five unrelated families. As discussed below, De Franco and Lytrivi et al. (20) also employed state-of-the-art laboratory experiments demonstrating the adverse functional impact of loss of YIPF5 function on pancreatic $\beta$ cell function in human-derived cell-based models.

\section{Endoplasmic reticulum stress}

Yip1p was discovered in Saccharomyces cerevisiae using a two-hybrid system with two Rab-like GTPases as bait (Ypt1 and Ypt31) (25). In a follow-up two-hybrid screen, Yif1p was discovered as a Yip1p-interacting protein (26). Functional studies demonstrated that Yip1p and Yif1p form a heterodimer, which plays a critical role in vesicle trafficking from the endoplasmic reticulum (ER) to the cis-Golgi; Rablike proteins play a downstream role in the process (26). Seven members of the Yip1 domain family have been identified in humans: YIPF1, YIPF2, YIPF3, YIPF4, YIPF5, YIPF6, and YIPF7 (27). YIPF5 is located in the early Golgi intermediate compartment (ERGIC) and is viewed as the ortholog of yeast Yip1p (28). YIPF5-containing complexes are believed to play a critical role in trafficking of COPII-coated vesicles from the ER to the cis-Golgi (27). Other YIPF family members are found in characteristic locations in the ER or Golgi and are also believed to function in vesicle transport $(27,29)$.

Proinsulin is synthesized by ribosomes in the rough ER and transported through the Golgi where it is packaged into secretory granules. Proinsulin undergoes proteolytic processing within secretory granules and is further transformed into mature insulin molecules (30). If transport from ER to Golgi were blocked, uncleaved proinsulin would accumulate within the ER with a consequent decrease in production of mature insulin molecules. Indeed, this was precisely the cellular phenotype that was observed when YIPF5 was knocked out in human embryonic stem cells that were subsequently differentiated into pancreatic $\beta$ cells (20). A qualitatively similar, but quantitatively milder, phenotype was seen when a pathogenic mutation (Ile98Ser) was knocked into the YIPF5 gene. Loss of YIPF5 function also induced ER stress and activated the unfolded protein response as demonstrated by increased BiP mRNA. In some respects, this phenotype is similar to that of the Akita mouse, which harbors a mutation (Cys96Tyr) in the insulin-2 gene that causes misfolding of proinsulin, thereby inducing ER stress as reflected by an increase in BiP mRNA (31).

De Franco and Lytrivi et al. (20) also investigated two other cell-based models. siRNA-mediated knockdown of YIPF5 mRNA increased vulnerability of EndoC$\beta \mathrm{H} 1$ cells to apoptosis induced by ER stressors (brefeldin A and thapsigargin). In 
addition, PBMCs from two siblings (both homozygous for the Ile98Ser mutation) were reprogrammed into induced pluripotent stem cells (iPSCs), then differentiated into a pancreatic $\beta$ cell phenotype. As compared with cells derived from healthy control subjects, the patients' cells were characterized by fewer insulin-positive cells and relatively more glucagon-positive cells. This difference was reversed when CRISPR/Cpf1 technology was applied to correct the mutation in patients' iPSCs. Viability of patient-derived $\beta$ cells appears to be lower than for $\beta$ cells derived from healthy controls, and the patient-derived $\beta$ cells were more vulnerable to apoptosis induced by ER stressors (brefeldin A, thapsigargin, and tunicamycin) (20). Further research will be required to elucidate the relative importance of these pathophysiological mechanisms in mediating the various aspects of the disease phenotype - both in pancreatic $\beta$ cells and in the brain.

\section{Conclusions and clinical implications}

Based on the observed pathophysiology associated with YIPF5 mutations, we hypothesize that an insulin sensitizer such as pioglitazone might improve glycemic control while decreasing the demand for insulin secretion, thereby alleviating ER stress and potentially improving $\beta$ cell health. Of course, even if pioglitazone were effective at improving glycemic control and $\beta$ cell health, we predict that this approach would not address important disease manifestations in the brain.

The publication by De Franco and Lytrivi et al. (20) illustrates the value of a biobank containing DNA from well-characterized patients with rare and atypical forms of diabetes, which enabled rapid identification of five families with mutation in YIPF5. Similar registries and biobanks have yielded important scientific insights into many rare diseases. Recognizing the importance of this approach, the National Institute of Diabetes and Digestive and Kidney Diseases (NIDDK) has recently funded the Rare and Atypical Diabetes Network (RADIANT, https:// www.atypicaldiabetesnetwork.org), a consortium to identify, study, and facilitate research on rare and atypical forms of diabetes to provide insights into the heterogeneity of human diabetes. The existence of such a network will also facilitate the ability of US-based researchers to participate in international collaborations. Such collaborative efforts will greatly accelerate the transformation of patient care through a precision medicine strategy.

\section{Acknowledgments}

TIP acknowledges support from research grants funded by the Eunice Kennedy Shriver National Institute of Child Health and Human Development (NICHD) (U24HD093486), the National Human Genome Research Institute (NHGRI) (U01HG007775), the NIDDK (U54DK1181612 and P30DK072488), and the Regeneron Genetics Center. SIT acknowledges support from research grants funded by NIDDK (T32DK098107, R01DK118942, and P30DK072488).

Address correspondence to: Toni I. Pollin, University of Maryland School of Medicine, HSF-III, Room 4040, 670 West Baltimore Street, Baltimore, Maryland 21201, USA. Email: tpollin@som.umaryland. edu. Or to: Simeon I. Taylor, University of Maryland School of Medicine, HSF-III, Room 4182, 670 West Baltimore Street, Baltimore, Maryland 21201, USA. Email: staylor2@som.umaryland.edu.

1. Chung WK, et al. Precision medicine in diabetes: A consensus report from the American Diabetes Association (ADA) and the European Association for the Study of Diabetes (EASD). Diabetes Care. 2020;43(7): 1617-1635.

2. Tattersall RB. Mild familial diabetes with dominant inheritance. QJMed.1974;43(170):339-357.

3. Tattersall RB, Fajans SS. A difference between the inheritance of classical juvenile-onset and maturity-onset type diabetes of young people. Diabetes. 1975;24(1):44-53.

4 . Vionnet $\mathrm{N}$, et al. Nonsense mutation in the glucokinase gene causes early-onset noninsulin-dependent diabetes mellitus. Nature. 1992;356(6371):721-722.

5. Yamagata K, et al. Mutations in the hepatocyte nuclear factor-4alpha gene in maturityonset diabetes of the young (MODY1). Nature. 1996;384(6608):458-460.

6. Yamagata K, et al. Mutations in the hepatocyte nuclear factor-1alpha gene in maturityonset diabetes of the young (MODY3). Nature. 1996;384(6608):455-458.

7. Berardinelli W. An undiagnosed endocrinometabolic syndrome: report of 2 cases. J Clin Endocrinol Metab. 1954;14(2):193-204.

8. Seip M. Lipodystrophy and gigantism with associated endocrine manifestations. A new diencephalic syndrome? Acta Paediatr. 1959;48:555-574.
9. Agarwal AK, et al. AGPAT2 is mutated in congenital generalized lipodystrophy linked to chromosome 9q34. Nat Genet. 2002;31(1):21-23.

10. Magré J, et al. Identification of the gene altered in Berardinelli-Seip congenital lipodystrophy on chromosome 11q13. Nat Genet. 2001;28(4):365-370.

11. Barnes ND, Palumbo PJ, Hayles AB, Folgar H. Insulin resistance, skin changes, and virilization: a recessively inherited syndrome possibly due to pineal gland dysfunction. Diabetologia. 1974;10(4):285-289.

12. Kahn CR, et al. The syndromes of insulin resistance and acanthosis nigricans. Insulin-receptor disorders in man. N Engl J Med. 1976;294(14):739-745.

13. Kadowaki T, et al. Two mutant alleles of the insulin receptor gene in a patient with extreme insulin resistance. Science. 1988;240(4853):787-790.

14. Yoshimasa Y, et al. Insulin-resistant diabetes due to a point mutation that prevents insulin proreceptor processing. Science. 1988;240(4853):784-787.

15. Accili D, et al. A mutation in the insulin receptor gene that impairs transport of the receptor to the plasma membrane and causes insulin-resistant diabetes. ЕMBO J. 1989;8(9):2509-2517.

16. Hattersley AT, Patel KA. Precision diabetes: learning from monogenic diabetes. Diabetologia. 2017;60(5):769-777.

17. Shepherd M, Pearson ER, Houghton J, Salt G, Ellard S, Hattersley AT. No deterioration in glycemic control in HNF-1alpha maturity-onset diabetes of the young following transfer from long-term insulin to sulphonylureas. Diabetes Care. 2003;26(11):3191-3192.

18. Gloyn AL, et al. Activating mutations in the gene encoding the ATP-sensitive potassium-channel subunit Kir6.2 and permanent neonatal diabetes. N Engl J Med. 2004;350(18):1838-1849.

19. Pearson ER, et al. Switching from insulin to oral sulfonylureas in patients with diabetes due to Kir6.2 mutations. N Engl J Med. 2006;355(5):467-477.

20. De Franco E, et al. YIPF5 mutations cause neonatal diabetes and microcephaly through endoplasmic reticulum stress. J Clin Invest. 2020;130(12):6338-6353.

21. Wildin RS, et al. X-linked neonatal diabetes mellitus, enteropathy and endocrinopathy syndrome is the human equivalent of mouse scurfy. Nat Genet. 2001;27(1):18-20.

22. Oral EA, et al. Leptin-replacement therapy for lipodystrophy. NEnglJMed.2002;346(8):570-578.

23. Richards $\mathrm{S}$, et al. Standards and guidelines for the interpretation of sequence variants: a joint consensus recommendation of the American College of Medical Genetics and Genomics and the Association for Molecular Pathology. Genet Med. 2015;17(5):405-424.

24. Rivera-Muñoz EA, et al. ClinGen Variant Curation Expert Panel experiences and standardized processes for disease and gene-level specification of the ACMG/AMP guidelines for sequence variant interpretation. Hum Mutat. 2018;39(11):1614-1622.

25. Yang X, Matern HT, Gallwitz D. Specific binding to a novel and essential Golgi membrane protein (Yip1p) functionally links the 
transport GTPases Ypt1p and Ypt31p. EMBO J. 1998;17(17):4954-4963.

26. Matern H, Yang X, Andrulis E, Sternglanz R, Trepte HH, Gallwitz D. A novel Golgi membrane protein is part of a GTPase-binding protein complex involved in vesicle targeting. $E M B O \mathrm{~J}$. 2000;19(17):4485-4492.

27. Shaik S, Pandey H, Thirumalasetti SK, Nakamura N. Characteristics and functions of the Yip1 domain family (YIPF), multi-span transmembrane proteins mainly localized to the Golgi apparatus. Front Cell Dev Biol. 2019;7:130.

28. Tang BL, et al. A membrane protein enriched in endoplasmic reticulum exit sites interacts with COPII. J Biol Chem. 2001;276(43):40008-40017.

29. Kranjc T, Dempsey E, Cagney G, Nakamura N, Shields DC, Simpson JC. Functional characterisation of the YIPF protein family in mammalian cells. Histochem Cell Biol. 2017;147(4):439-451.

30. Liu M, Wright J, Guo H, Xiong Y, Arvan P. Proinsulin entry and transit through the endoplasmic reticulum in pancreatic beta cells. Vitam Horm. 2014;95:35-62.

31. Izumi T, Yokota-Hashimoto H, Zhao S, Wang J, Halban PA, Takeuchi T. Dominant negative pathogenesis by mutant proinsulin in the Akita diabetic mouse. Diabetes. 2003;52(2):409-416. 